\title{
Absence of human herpesvirus-8 DNA in Kaposi's sarcoma following postmastectomy lymphoedema
}

\author{
du Plessis DG, Schneider JW, Treurnicht FK, Engelbrecht S\& Janse van Rensburg E \\ ${ }^{0}$ Departments of Anatomical Pathology \& Virology, University of Stellenbosch and Tygerberg Hospital, \\ Tygerberg, South Africa
}

Sir: A variety of tumours, the most common example being angiosarcoma (Stewart-Treves syndrome), have been described in extremities affected by chronic lymphoedema. ${ }^{1}$ Lymphoedema may follow, coincide or precede classic or HIV-associated Kaposi's sarcoma (KS). ${ }^{2,3}$ However, the development of KS in limbs with primary or secondary chronic lymphoedema in patients with no risk factors for KS is very rare.

Only two cases of KS arising in a chronic lymphoedematous arm following radical mastectomy have been reported. ${ }^{3}$ We present the clinicopathological features of a third example, including an investigation into the presence of associated human herpesvirus-8 (HHV-8) in this situation. A 67-year-old woman without predisposing factors for KS underwent a radical mastectomy and axillary lymph node resection for infiltrating duct carcinoma of the right breast, followed by a course of local radiotherapy. She remained tumour free with moderate, persistent lymphoedema of the right arm for 16 years until she presented with a small intradermal nodule in the right elbow region. Histology revealed a spindle cell tumour consistent with nodular KS (Figure 1).

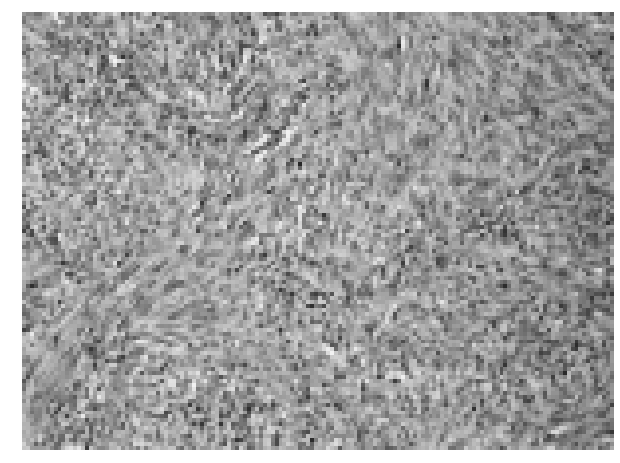

Figure 1. The initial biopsy showed nodular KS consisting of short fascicles of spindle cells interrupted by slitlike spaces containing red blood cells $(H \& E)$

Immunohistochemistry showed positive staining of tumour cells for CD34 and CD31 using a routine strepavidin-biotin complex (ABC) method and monoclonal antibodies obtained from Dako. Three years later 'eczematous' lesions were noted in the same area. A biopsy showed features consistent with patch phase KS (Figure 2). 


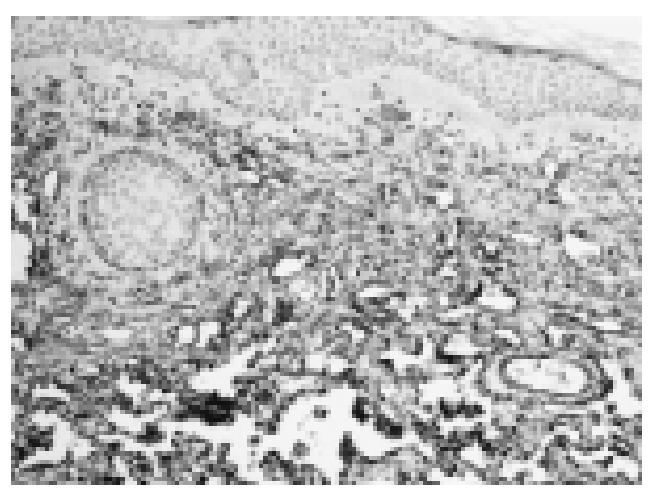

Figure 2. Patch-phase KS lesion biopsied 3 years later. CD31 immunostaining highlights irregular angular vascular spaces lined by a single layer of endothelial cells without signi ${ }^{\circledR}$ cant nuclear atypia dissecting dermal collagen bundles and surrounding native dermal vessels and adnexal structures.

Apart from transient, limited exacerbation during a course of chemotherapy for biopsyproven metastatic breast carcinoma, the KS lesions have remained static over a 14-years follow-up period, some even showing regression.

Sections from formalin-fixed, paraffin-embedded tissue representative of the original breast carcinoma, normal breast, subsequent metastases, and all biopsies of KS lesions were submitted for the detection of HHV-8 DNA sequences. KS330 233 primers were used to amplify an HHV-8 specific 233-base pair sequence of open reading frame 26. The polymerase chain reaction (PCR) products were subsequently used with nested primers to amplify a 172-base pair sequence. Dilutions of the plasmid KS330Bam were included as positive controls and to determine the number of HHV-8 target molecules present. All the conditions and primers were used as described previously. ${ }^{4} \mathrm{HHV}-8$ specific sequences were not detected in any of the specimens.

Although HHV-8 is strongly associated with KS in systemic immune-suppressed patients, a similar relationship between lymphoedema with regional immune-suppression and HHV-8 remains to be proven. Recently Cerri et al. failed to demonstrate HHV-8 DNA in a lymphangiosarcoma of the pubic region that developed in lymphoedematous tissue due to congenital lymphoedema. ${ }^{5}$ The absence of HHV-8 DNA in occasional KS lesions is well documented. This observation may be due to technical factors, incorrect diagnosis, extremely low copy numbers of HHV-8 in the KS lesions, or viral mutations that escape detection by the $\mathrm{KS}_{330}{ }_{233}$ primer. $^{6}$ However, it remains possible that factors other than HHV-8 may stimulate endothelial cell proliferation and induce anti-apoptotic factors in KS.

Rare cases of localized KS that develop in chronically lymphoedematous limbs of patients who do not have other clinico-epidemiological risk factors for KS, suggest that chronic lymphoedema may be an additional condition predisposing to the development of KS. The demonstration of a reduced delayed type hypersensitivity response in such limbs, has prompted the hypothesis that a regional immune defect limited to areas of localized lymphoedema might also involve the mechanisms of antineoplastic immune surveillance, thereby facilitating the development of certain tumours in those areas. ${ }^{2}$ Additional studies are 
necessary to determine whether KS in lymphoedematous tissue is less frequently associated with HHV-8 than other KS variants.

\section{References}

1. D'amore ESG, Wick MR, Geisinger KR, Frizzera G . Primary malignant lymphoma arising in postmastectomy lymphedema. Another facet of the Stewart-Treves syndrome. Am. J. Surg. Pathol. 1990; 14; 456463.

2.Ruocco V, Astarita C, Guerrera V et al. Kaposi's sarcoma on a lymphedematous immunocompromised limb. Int. J. Dermatol. 1984; 23; 5660.

3.Ron IG, Amir G, Marmur S, Chaitchik S, Inbar MJ . Kaposi's sarcoma on a lymphedematous arm after mastectomy. Am. J. Clin. Oncol. 1996; 19; 8790.

4. Engelbrecht S, Treurnicht FK, Schneider JW et al. Detection of Human Herpes virus 8 DNA and sequence polymorphism in classical, epidemic, and iatrogenic Kaposi's sarcoma in South Africa. J. Med. Virol. 1997; 52; 168172.

5. Cerri A, Gianni C, Pizzuto M, Moneghini L, Crosti C . Lymphangiosarcoma of the pubic region: a rare complication arising in congenital non-hereditary lymphedema. Eur. J. Dermatol. 1998; 8; 511514.

6. Noel J-C, Hermans P, Andre J et al. Herpesvirus-like DNA sequences and Kaposi's sarcoma. Relationship with epidemiology, clinical spectrum, and histological features. Cancer 1996; 77; 21322136. 